\title{
Macrophages suppress T cell responses and arthritis development in mice by producing reactive oxygen species
}

\author{
Kyra A. Gelderman, ${ }^{1}$ Malin Hultqvist, ${ }^{1}$ Angela Pizzolla, ${ }^{1}$ Ming Zhao, ${ }^{1}$ \\ Kutty Selva Nandakumar, ${ }^{1}$ Ragnar Mattsson, ${ }^{1}$ and Rikard Holmdahl1,2
}

${ }^{1}$ Section of Medical Inflammation Research, Lund University, Lund, Sweden. ${ }^{2}$ MediCity Research Laboratory, Turku University, Turku, Finland.

\begin{abstract}
Reduced capacity to produce ROS increases the severity of $\mathrm{T}$ cell-dependent arthritis in both mice and rats with polymorphisms in neutrophil cytosolic factor 1 (Ncf1) (p47phox). Since T cells cannot exert oxidative burst, we hypothesized that $T$ cell responsiveness is downregulated by ROS produced by APCs. Macrophages have the highest burst capacity among APCs, so to study the effect of macrophage ROS on T cell activation, we developed transgenic mice expressing functional Ncf1 restricted to macrophages. Macrophage-restricted expression of functional Ncf1 restored arthritis resistance to the level of that of wild-type mice in a collageninduced arthritis model but not in a $T$ cell-independent anti-collagen antibody-induced arthritis model. $T$ cell activation was downregulated and skewed toward Th2 in transgenic mice. In vitro, IL-2 production and $T$ cell proliferation were suppressed by macrophage ROS, irrespective of $T$ cell origin. IFN- $\gamma$ production, however, was independent of macrophage ROS but dependent on $T$ cell origin. These effects were antigen dependent but not restricted to collagen type II. In conclusion, macrophage-derived ROS play a role in T cell selection, maturation, and differentiation, and also a suppressive role in $T$ cell activation, and thereby mediate protection against autoimmune diseases like arthritis.
\end{abstract}

\section{Introduction}

RA is an autoimmune disease with a prevalence of around $1 \%(1)$. In spite of this high incidence and its severe phenotype, RA cannot be cured yet. The cause of RA is unknown although it has been estimated that it is influenced by genetic factors in up to $60 \%$ of cases (2). Therefore, identification of genes that predispose for RA will shed light on immunological pathways that play a role in disease initiation and might help to develop more effective therapeutics (3). One such genetic polymorphism that we previously identified in congenic rats is located in the neutrophil cytosolic factor 1 (Ncf1) gene, a finding confirmed in mice $(4,5)$. Ncf1 (p47phox) encodes one of the activating proteins in the phagocytic NADPH oxidase complex, and the genetic variants found in rats and mice lead to a lower capacity to exert oxidative burst. Interestingly, collagen-induced arthritis (CIA) in mice and rats and pristane-induced arthritis (PIA) in rats were shown to be more severe and of higher incidence in animals with $N c f 1$ variants associated with lower burst capacity. Also, in a model for multiple sclerosis in mice (experimental autoimmune encephalomyelitis), it was observed that Ncf1 mutant mice developed more severe disease, suggesting that ROS have a general immune suppressive effect (4).

The NADPH oxidase complex produces ROS and is mainly expressed in phagocytic cells. The ability to release ROS via oxidative burst represents an important component of antimicrobial defense mechanisms in the host (6). Mice and humans deficient in one of the protein members of this complex develop chronic granulomatous

Nonstandard abbreviations used: CAIA, collagen antibody-induced arthritis; CIA, collagen-induced arthritis; CII, collagen type II; ConA, concanavalin A; DHR123, dihydrorhodamine 123; lathCII, lathyritic CII; MN, macrophage Ncf1; Ncf1, neutrophil cytosolic factor 1; PIA, pristane-induced arthritis; p-macrophage, peritoneal macrophage; PPD, purified protein derivative; $-\mathrm{SH}$, thiol group.

Conflict of interest: The authors have declared that no conflict of interest exists. Citation for this article: J. Clin. Invest. 117:3020-3028 (2007). doi:10.1172/JCI31935. disease (CGD) $(7,8)$. Although required to combat infections, ROS can be harmful in the effector phase of inflammatory diseases, like RA. Many studies have addressed the role of ROS in arthritis, but they mostly investigated ROS production in the joints (9). These reports conclude that the ability of neutrophils in the joints of RA patients to produce ROS is higher as compared with healthy controls, probably as a result of the local inflammatory reaction. Increased ROS levels may subsequently mediate local damage and further immune activation (9). It is, however, important to discriminate between harmful massive burst induced by pathogens or during the effector phase of inflammatory responses and the ROS produced as mediators of immune regulation. ROS may also regulate intracellular signaling and lymphocyte activity in the initiation phase of the immune response $(10,11)$. We showed that the mutations in $N c f 1$ exert their effect already in the priming phase of the immune response and provided evidence that the reduced capacity to produce ROS leads to enhanced arthritis via activation of autoreactive T cells $(4,5,12)$. Since we could not detect oxidative burst or $\mathrm{Ncf1}$ expression in T cells $(12,13)$, it is likely that other cells act as regulators of $\mathrm{T}$ cell activation by producing ROS. T cells have close contact with APCs that are able to produce ROS via the NADPH oxidase complex. We hypothesized that $\mathrm{T}$ cell responses in animals with arthritis-associated $N c f 1$ alleles are increased because of defective ROS production in APCs $(14,15)$.

To test this hypothesis, we have investigated the role of ROS produced by macrophages, being the APCs with the highest burst capacity. The mice with mutated $N c f 1$ provided an optimal model to address this issue, especially since they are genetically clean and have only 1 nucleotide difference compared with the wild type. We show that expression of functional $N c f 1$ restricted to macrophages suppressed arthritis development via diminishing $\mathrm{T}$ cell activation, which was mediated in an antigen-dependent fashion. These data indicate a new role for macrophage-derived ROS in suppressing immune activation and preventing autoimmunity. 
A
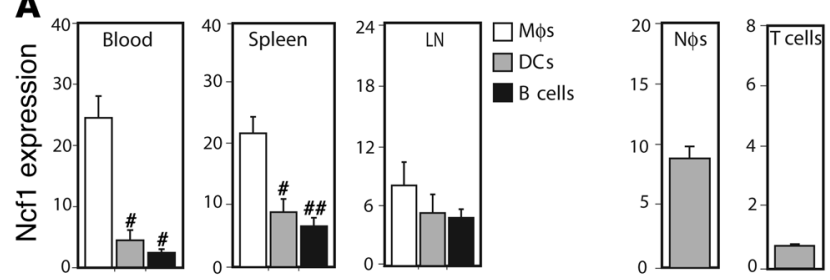

B
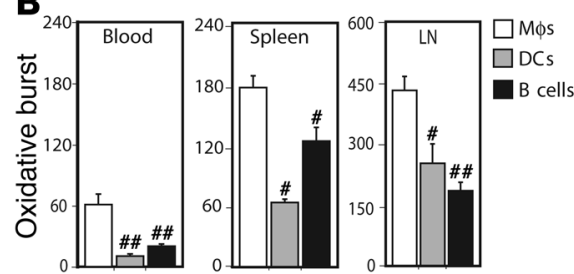

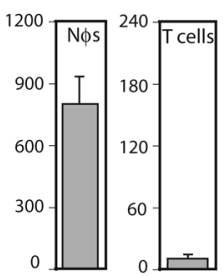

Figure 1

Macrophages are the APCs with the highest Ncf1 expression and oxidative burst capacity. Expression levels of Ncf1 in APCs (A) and their ability to exert oxidative burst $(B)$ were determined in cells from naive B10.Q mice. Macrophages ( $\left.\mathrm{M} \phi: \mathrm{F} 4 / 80^{+} \mathrm{CD} 11 \mathrm{c}^{-}\right)$showed significantly higher levels of $\mathrm{Ncf} 1$ expression and oxidative burst induced by PMA as compared with DCs (F4/80-CD11 $\left.\mathrm{C}^{+}\right)$and $\mathrm{B}$ cells $\left(\mathrm{B} 220^{+} \mathrm{CD} 11 \mathrm{~b}^{-}\right)$in blood, spleen, and inguinal LNs. Means \pm SEM are shown of 6 animals per group. Asterisks indicate significantly lower expression or burst as compared with macrophages: ${ }^{\#} P<0.05 ;{ }^{\#} P<0.01$. Ncf1 expression and burst of blood neutrophils ( $\left.\mathrm{N} \phi: \mathrm{Gr}^{+}{ }^{+} \mathrm{F} 4 / 80^{-}\right)$and $\mathrm{T}$ cells ( $\mathrm{T}$ : $\left.\mathrm{CD}^{+}{ }^{+} \mathrm{CD} 3^{+}\right)$are shown as positive and negative controls, respectively.

\section{Results}

Macrophages are the APCs with the highest ROS production. First, we identified which APC type had the highest level of Ncf1 expression and the highest capacity to exert oxidative burst. Different lymphoid organs were taken from naive B10.Q mice and analyzed for Ncf1 expression and burst in different APCs by flow cytometry. Ncf1 was expressed at higher levels in monocytes/macrophages (referred to as macrophages; $\left.\mathrm{F} 4 / 80^{+} \mathrm{CD} 11 \mathrm{c}^{-}\right)$as compared with $\mathrm{DCs}\left(\mathrm{CD} 11 \mathrm{c}^{+} \mathrm{F} 4 / 80^{-}\right)$and $\mathrm{B}$ cells $\left(\mathrm{B} 220^{+} \mathrm{CD} 11 \mathrm{~b}^{-}\right)$in blood, spleen, and inguinal LNs (Figure 1A) and also in bone marrow and thymus (not shown). Upon stimulation with PMA, macrophages were most efficient in producing ROS compared with the other cell types, as measured with dihydrorhodamine 123 (DHR123) (Figure 1B). The same was observed in bone marrow (not shown). Blood neutrophils and T cells are shown as positive and negative controls, respectively. Levels of DHR and Ncf1 staining in $\mathrm{CD}^{+} \mathrm{CD}^{+}$cells were similar to control conditions without PMA or without antibodies to $\mathrm{Ncf1}$, respectively.

Transgenic mice have higher Ncf1 expression and oxidative burst in macrophages only. Since macrophages had the highest capacity to burst as compared with DCs and B cells, macrophages might be important APCs in regulating $T$ cell responses via ROS. To investigate the role of Ncf1 in macrophages specifically, a transgenic mouse was developed, expressing functional Ncf1 restricted to macrophages on an Ncf1 mutant B10.Q background using a human CD68 promoter (B10.Q ${ }^{M N}$ transgenic mice; MN, macrophage Ncf1) (16). The Ncf1 mutation, originally on a C57BL/6J-m $\mathrm{m}^{+/+} \operatorname{Lepr}^{\mathrm{db}}$ background, was backcrossed to B10.Q for more than 12 generations, and we could not identify any remaining B6-specific fragments through informative microsatellites in the congenic mouse $(4,17)$. The mutation in Ncf1 affects splicing and leads to expression of low levels of truncated forms of $\mathrm{Ncf} 1$ protein that were not detectable with the FACS

staining used (17). To confirm functional expression of Ncf1 due to the transgene, Ncf1 expression was determined by flow cytometry in mice with all genotypes for $N c f 1$ and positive or negative for the transgene. In the $N c f 1$ mutated transgenic mice $\left(N c f 1^{* / *} \mathrm{MN}^{+}\right)$, significantly higher levels of Ncf1 were detected in macrophages $\left(\mathrm{F} 4 / 80^{+} \mathrm{CD} 11 \mathrm{c}^{-}\right)$from spleen as compared with transgene-negative mice $\left(N c f 1^{* / *} \mathrm{MN}^{-}\right)$, reaching levels comparable to those in $\mathrm{Ncf1}$ heterozygous $\left(\mathrm{Ncf1}^{+/ *}\right)$ mice. This difference in expression was neither observed in other spleen APCs (DCs [CD11 $\left.\mathrm{c}^{+} \mathrm{F} 4 / 80^{-}\right]$and B cells $\left[\mathrm{B} 220^{+} \mathrm{CD}_{\left.\left.11 \mathrm{~b}^{-}\right]\right)}\right.$nor in $\mathrm{T}$ cells, indicating macrophage-specific expression (Figure $2 \mathrm{~A})$. In neutrophils $\left(\mathrm{Gr} 1^{+} \mathrm{F} 4 / 80^{-}\right)$, no differences in Ncf1 expression or burst between transgenic and nontransgenic mice were observed either, indicating that the CD68 promoter did not act in neutrophils (not shown). Functionality of Ncf1 in macrophages from $\mathrm{Ncfl}^{* * *}$ transgenic mice was confirmed by measuring ROS production in spleen macrophages after stimulation with PMA, showing that macrophages from mice expressing the transgene $\left(\mathrm{MN}^{+}\right)$were indeed able to exert oxidative burst in contrast to those from transgene-negative mice $\left(\mathrm{MN}^{-}\right)$(Figure $\left.2 \mathrm{~B}\right)$. In $\mathrm{Ncf1}^{+/ *}$ mice, a significant difference in $\mathrm{Ncf1}$ expression between $\mathrm{MN}^{-}$and $\mathrm{MN}^{+}$mice was observed as well, although this did not translate into a significant difference in burst, which might be due to differences in sensitivity or variation in the assays used. In other organs and in blood, similar results were obtained (not shown).

ROS produced by macrophages protect against arthritis. To determine whether expression of functional Ncf1 restricted to macrophages had an ameliorating effect on arthritis development, arthritis was induced in littermates representing the 6 groups by immunization with collagen type II (CII) to induce CIA. Ncf1 mutant transgenic mice $\left(N c f 1^{* / *} \mathrm{MN}^{+}\right)$expressing functional $\mathrm{Ncf} 1$ on macrophages only developed significantly less severe arthritis than the Ncf1 mutant, nontransgenic controls $\left(N c f 1^{* / *} \mathrm{MN}^{-}\right)$(Figure 3A). This protective effect was not only observed in the homozygous mutant mice but also in the heterozygous $\left(\mathrm{Ncf1}^{+/ *}\right)$ mice, arguing for a dose-dependent effect of ROS produced by macrophages on $\mathrm{T}$ cell activation. The disease pattern in $N c f 1^{* / *} \mathrm{MN}^{+}$mice was similar to that in $\mathrm{Ncf1}^{+/ *} \mathrm{MN}^{-}$mice and only increased in severity after boost at day 35 whereas $N c f 1^{* / *} \mathrm{MN}^{-}$mice developed severe arthritis soon after immunization, before boost. The difference in severity due to transgenic expression of $\mathrm{Ncf1}$ in macrophages was not observed in the $\mathrm{Ncf1}$ wild-type $\left(\mathrm{Ncf1}^{+/+}\right)$mice, indicating that the effect of the transgene was not dependent on interference with other genes.

We showed before that $N c f 1^{+/ *}$ and $\mathrm{Ncf1}^{+/+}$mice produced lower levels of anti-CII Ab as compared with the $N c f 1^{* / *}$ mice (4). To determine whether this difference was also present between $\mathrm{Ncfl}^{* / *} \mathrm{MN}^{-}$and $\mathrm{Ncf1} 1^{* / *} \mathrm{MN}^{+}$mice, serum was obtained at days 10 , 42 , and 89 after immunization and assayed for presence of antibodies of different isotypes reactive with CII. As shown in Figure 3B, the levels of total anti-CII IgG were significantly lower in $\mathrm{Ncfl}^{*}$ ${ }^{*} \mathrm{MN}^{+}$mice as compared with the $N c f 1^{* * *} \mathrm{MN}^{-}$mice at day 42 and day 89 . Already at day $10, N c f 1^{* / *}$ mice had produced more anti-CII IgG as compared with $\mathrm{Ncf1}^{+/ *}$ or $\mathrm{Ncf1}^{+/+}$mice, coinciding with the earlier disease onset, although total levels were low compared with the other days. Comparable patterns were observed for the specific subclasses IgG1, IgG2a, and IgG2b, with higher levels of IgG subclasses in $\mathrm{Ncfl}^{*}{ }^{* *} \mathrm{MN}^{-}$mice at day 43 and less pronounced differences on day 89 (Supplemental Figure 1, A-C; supplemental material available online with this article; doi:10.1172/JCI31935DS1). The levels of total IgG in serum did not differ between the different genotypes, excluding a direct effect of the mutation in Ncf1 on 

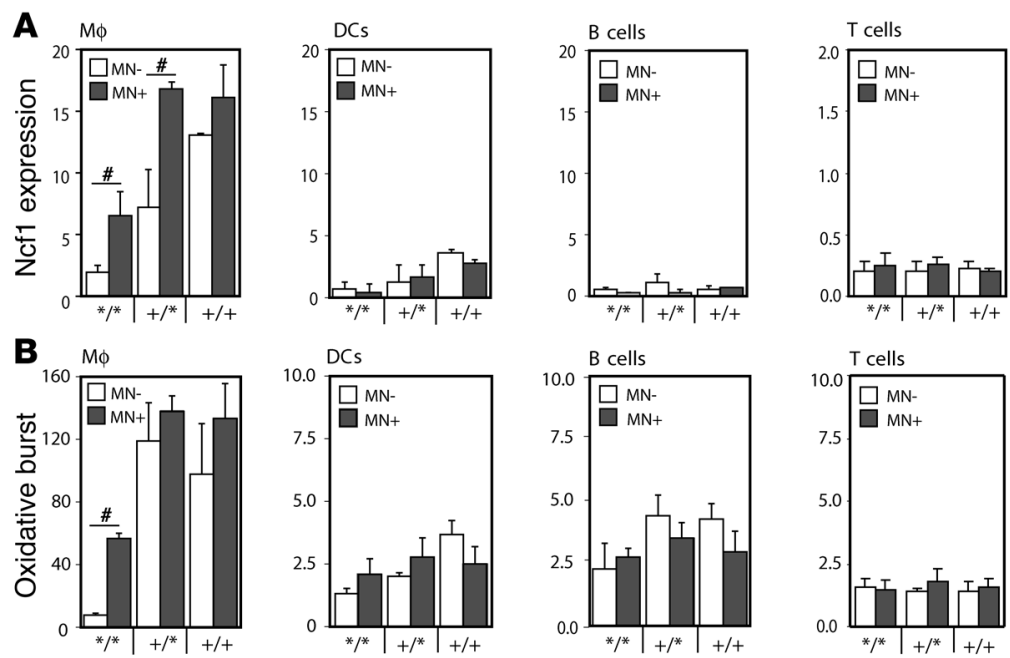

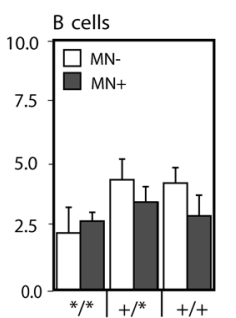

Figure 2

Transgenic mice show increased Ncf1 expression and burst by macrophages only. (A) Expression levels of Ncf1 were determined in $\mathrm{B} 10 . \mathrm{Q}^{M N}$ transgenic mice, expressing functional Ncf1 under control of the human CD68 promoter on an Ncf1 mutant $\left({ }^{*} / *\right)$, heterozygous $\left(+/^{*}\right)$, or wild-type $(+/+)$ background. Spleen macrophages (F4/80+CD11C-) express significantly higher levels of Ncf1 when positive for the transgene (black bars: $\mathrm{MN}^{+}$) as compared with transgene-negative mice (white bars: $\mathrm{MN}^{-}$). This difference in Ncf1 expression was not observed in DCs $\left(\mathrm{F} 4 / 80^{-} \mathrm{CD} 11 \mathrm{C}^{+}\right)$, B cells $\left(\mathrm{B} 220^{+} \mathrm{CD} 11 \mathrm{~b}^{-}\right)$, or $\mathrm{CD}^{+} \mathrm{T}$ cells $\left(\mathrm{CD} 3^{+} \mathrm{CD} 4^{+}\right)$. (B) Accordingly, macrophages from Ncf1 ${ }^{* / *}$ transgene-positive $\left(\mathrm{MN}^{+}\right)$mice were able to exert oxidative burst that was significantly higher than in transgenenegative $\left(\mathrm{MN}^{-}\right)$mice. This difference was not observed in DCs, B cells, or T cells. Mean \pm SEM of 4 mice are shown. ${ }^{P}<0.05$.
B cell function (Supplemental Figure 1D). These results suggest differences in $\mathrm{T}$ cell help to $\mathrm{B}$ cells as a result of a lower ROS production in macrophages.

Ncf1 in macrophages is not important during the effector phase of arthritis. The observed difference in anti-CII Ab levels might be responsible for the observed difference in arthritis severity in transgenic versus nontransgenic mice. Although we have previously shown that arthritis in both Ncf1 mutant mice and rats is T cell mediated, we wanted to exclude the possibility that functional Ncf1 on macrophages mediated its arthritis-ameliorating effect in the joint during the effector phase, via, e.g., increased levels of anti-CII $\mathrm{mAb}$ and subsequent complement activation and $\mathrm{FcR}$ interactions. The general belief is that ROS are harmful in the effector phase of arthritis and enhance joint destruction; therefore, one would expect increased disease severity in mice expressing functional $N c f 1$. To investigate this in a T cell-independent model, arthritis was induced with a cocktail of antibodies directed against 4 different epitopes on CII (C1, J1, $\mathrm{U} 1, \mathrm{C} 2$; collagen antibody-induced arthritis [CAIA]) (18). It was indeed observed that $N c f 1^{*}{ }^{*}$ mice had a tendency to develop less severe CAIA (Figure 4A) with lower incidence (Figure 4B) after LPS injection at day 7 , opposite to the pattern observed in CIA. Presence or absence of functional Ncf1 in macrophages did not result in different disease patterns, severity, or incidence. This indicates that Ncf1 in macrophages does not have its major regulatory effect on the effector phase of arthritis in the joints but rather operates through modifying the initiation phase.

Mutated Ncf1 in macrophages increases T cell reactivity. ROS as produced by macrophages might influence $\mathrm{T}$ cell response, resulting in the observed difference in arthritis severity and anti-CII IgG levels. To investigate the role of macrophage-derived ROS on $\mathrm{T}$ cell activation, we determined $T$ cell responses just after the priming phase but before disease onset. Spleen cells were isolated from mice with the different genotypes 10 days after immunization with pepsin-digested CII (CII) in CFA. Cell suspensions were restimulated in vitro with lathyritic CII (lathCII)
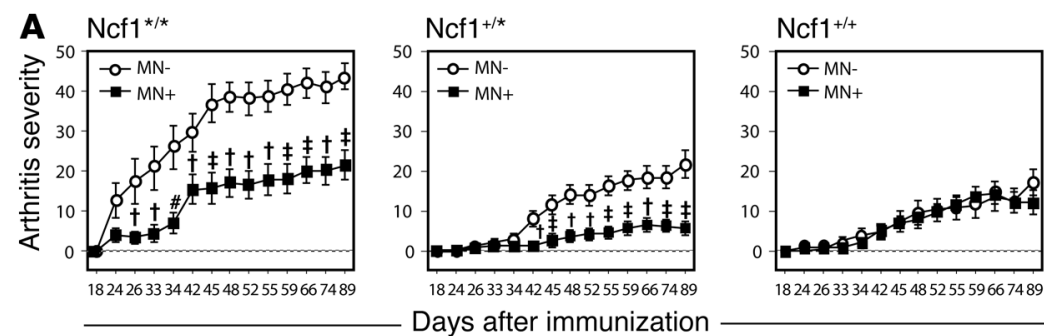

B
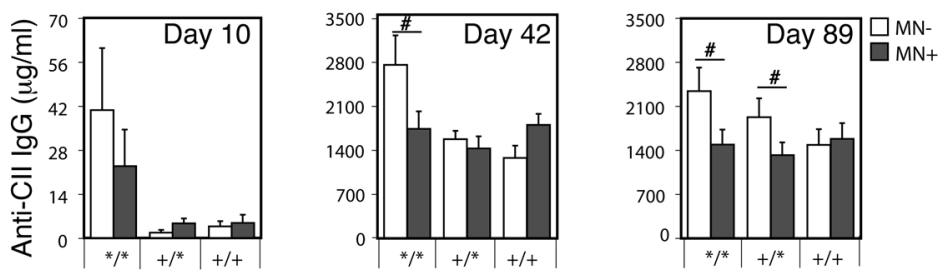

Figure 3

ROS production by macrophages decreases arthritis severity. (A) Mice expressing functional Ncf1 on macrophages only $\left(\mathrm{Ncf1}^{* / *} \mathrm{MN}^{+}\right.$: filled squares; $\left.n=23\right)$ developed significantly less severe CIA as compared with $\mathrm{Ncf1}^{* / *} \mathrm{MN}^{-}$(open circles; $n=15$ ) mice. After boost at day 35, a similar difference was also observed between Ncf1 heterozygous $\left(+/^{*}\right)$ mice with $(n=34)$ or without $(n=29)$ the transgene. No differences were observed in Ncf1 wild-type (+/+;n=21 and 27) mice. All groups were included in each experiment. Mean \pm SEM are shown of all mice, run in 2 different experiments with exactly the same setup, with the indicated total number of mice per group. ${ }^{P} P<0.05$; $\dagger P<0.005$; $¥ P<0.0005$. (B) Anti-Cll IgG levels were determined at 10,42 , and 89 days after immunization and were significantly lower in transgene-positive $\left(\mathrm{MN}^{+}\right) \mathrm{Ncf1}^{* / *}$ mice as compared with transgene-negative $\left(\mathrm{MN}^{-}\right) \mathrm{Ncf1}^{* / *}$ mice. Sera from the CIA experiments as shown in A were used, with similar numbers of mice as indicated there. Means \pm SEM are shown. 


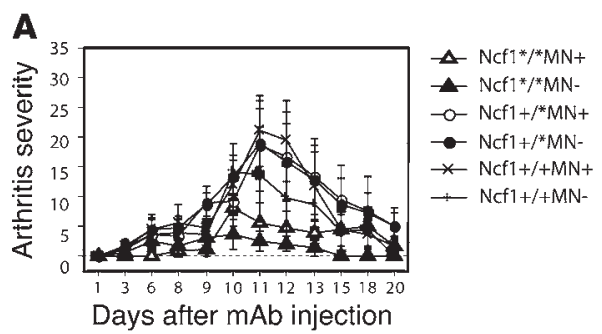

B

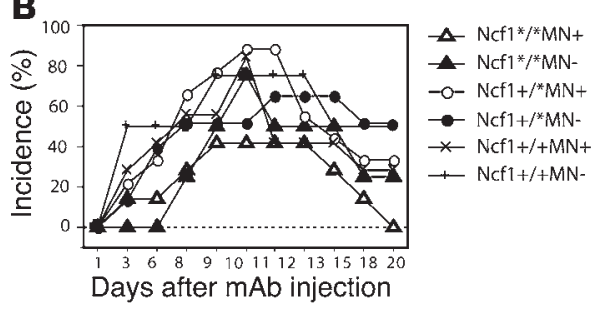

Figure 4

ROS produced by macrophages do not affect the inflammatory phase. CAIA was induced by injecting $4 \mathrm{mg}$ of a $4 \mathrm{mAb}$ cocktail reactive with CII i.v. into B10.Q ${ }^{M N}$ mice. LPS was injected 7 days later (day 7). Arthritis severity and incidence were determined over time. Mean \pm SEM are shown. No significant differences between transgene-positive or -negative mice were observed for either Ncf1 genotype $\left(\mathrm{Ncf1}^{* / *} \mathrm{MN}^{-}, n=7 ; \mathrm{MN}^{+}, n=6 ; \mathrm{Ncf}^{+l^{*}} \mathrm{MN}^{-}, n=9 ; \mathrm{MN}^{+}, n=8\right.$; $\left.\mathrm{Ncf}^{+/+} \mathrm{MN}^{-}, n=6 ; \mathrm{MN}^{+}, n=3\right)$.

determine whether this was dependent on increased activation or a higher frequency of activated T cells, IFN- $\gamma$ ELISPOT assays were conducted. It was shown that fewer $\mathrm{T}$ cells from $\mathrm{Ncf1} 1^{* / *} \mathrm{MN}^{+}$mice produced IFN- $\gamma$ as compared with $N c f 1^{* / *} \mathrm{MN}^{-}$mice when restimulated with lathCII (Figure 5B), indicative of decreased responsiveness of the $\mathrm{T}$ cells to $\mathrm{CII}$ as a result of ROS production by macrophages. In addition, the spot size was significantly smaller in $N c f 1^{* / *} \mathrm{MN}^{+}$ mice as compared with $\mathrm{Ncfl}_{1} / \mathrm{MN}^{-}$mice, indicating that the amount of IFN- $\gamma$ produced was also lower (not shown). Similar results were obtained when inguinal LN cells were used (not shown).

To exclude the possibility that the transgene affected antigen presentation by DCs, DCs were grown from bone marrow from naive mice under GM-CSF stimulation, and DC phenotype was confirmed by FACS (19). DCs were mixed with CII to allow uptake and processing and subsequently stimulated with LPS to induce maturation and upregulation of MHC class II and costimulatory molecules. In Figure $5 \mathrm{~A}$, it is shown that $\mathrm{T}$ cell responses did not differ between $N c f 1^{+/ *} \mathrm{MN}^{-}$and $\mathrm{Ncf1}^{+/ *} \mathrm{MN}^{+}$or $\mathrm{Ncf1}^{+/+} \mathrm{MN}^{-}$mice. For this reason, we only show $\mathrm{Ncf1}^{+/+} \mathrm{MN}^{-}$mice as controls in this and subsequent experiments. Mature DCs were coincubated with HCQ10 hybridoma T cells, specific for the glycosylated CII epitope 259-270 bound to $\mathrm{H} 2-\mathrm{A} q$, and IL-2 production was determined as a measure of $\mathrm{T}$ cell activation (20). It was shown that DCs from these mice induced comparable levels of $\mathrm{T}$ cell activation, regardless of presence or absence of the transgene (Figure 5C). As a control, macrophages were isolated from the peritoneum from naive mice and exposed to CII and HCQ10 T cells. Peritoneal macrophages ( $\mathrm{p}$-macrophages) from $\mathrm{Ncfl}^{*}{ }^{*} \mathrm{MN}^{-}$mice induced higher levels of IL-2 production as compared with those from $N c f 1^{* / *} \mathrm{MN}^{+}$mice, which were comparable to IL-2 production induced by $\mathrm{Ncfl}^{1^{+/}} \mathrm{MN}^{-}$macrophages (Figure 5D). No differences in IL-2 production were observed between genotypes in absence of CII (not shown). These data indicate that the transgene only acts in macrophages and excludes a role in DCs.
To determine whether the observed increased $\mathrm{T}$ cell response in $\mathrm{Ncf}_{1}{ }^{*}{ }^{*} \mathrm{MN}^{-}$mice was restricted to a specific antigen, mice were immunized with OVA instead of CII, and after 10 days, spleens and LNs were harvested. Single-cell suspensions were restimulated with OVA, and subsequently IL-2 (Figure 5E) and IFN- $\gamma$ (Figure 5F) production was determined by ELISA and ELISPOT, respectively. Similar patterns were observed as with CII. For spleen, the differences between $\mathrm{Ncfl}^{* / *} \mathrm{MN}^{-}$and $\mathrm{Ncfl}^{* / *} \mathrm{MN}^{+}$mice were not as big as in the CII experiments but were more pronounced when LNs were used. These data indicate that $\mathrm{T}$ cell responses against different antigens are lower when macrophages can produce ROS and are thus not restricted to CII.

Macrophages expressing functional Ncf1 suppress $T$ cell responses in an antigen-dependent way. To confirm dependence on antigen presentation and to determine whether the increased $\mathrm{T}$ cell activation as shown in Figure 5 was dependent on an increased activation status of the $\mathrm{T}$ cells or rather on an increased ability of macrophages to present antigen, the following experiments were performed. $\mathrm{CD}^{+}$ $\mathrm{T}$ cells were isolated from spleens from $N c f 1^{* / *} \mathrm{MN}^{-}, \mathrm{Ncfl}^{* / *} \mathrm{MN}^{+}$, and $\mathrm{Ncf1}^{+/+} \mathrm{MN}^{-}$mice, 10 days after immunization with CII. Macrophages were isolated from the peritoneum of naive mice with the same genotypes. Macrophages and T cells were coincubated in the 9 possible combinations while stimulated with CII, purified protein derivative (PPD), or no antigen. PPD is the immunogenic mycobacterial compound in CFA and is here used as a positive control. This setup allowed us to conclusively state whether the effect of macrophage ROS on $\mathrm{T}$ cell activation was mediated via antigen presentation or not. The results confirmed that $\mathrm{T}$ cells from mice lacking oxidative burst (due to mutated Ncf1) responded more vigorously in vitro than $\mathrm{T}$ cells from mice with functional burst only in macrophages or in all APC types (Figure 6A). This was independent of how activation was measured (by proliferation, IL-2, or IFN- $\gamma$ production). This indicates the processes of $\mathrm{T}$ cell selection, maturation, and differentiation in mice with mutated Ncf1 in macrophages are different in comparison to those in mice with functional Ncf1 in macrophages. However, when the effect of macrophages with functional burst on these $\mathrm{T}$ cells was addressed, it became clear that these macrophages suppressed the $\mathrm{T}$ cell response in vitro, as measured by proliferation and IL- 2 but not IFN- $\gamma$ production. This shows that ROS produced by macrophages also affect antigen presentation in vitro and confirms that macrophages suppress immune responses in vivo by producing ROS. That the IFN- $\gamma$ production was increased in all conditions with $N c f 1^{* / *} \mathrm{MN}^{-} \mathrm{T}$ cells, regardless of the origin of the macrophages, indicates that IFN- $\gamma$ production during presentation was not influenced by macrophages but only dependent on the origin of the T cells. Since the only difference between the mice from which the $\mathrm{T}$ cells originated was functional Ncf1 in macrophages, this argues for an educational effect of macrophage ROS earlier than during peripheral antigen presentation, e.g., in the spleen or thymus. Conditions stimulated with PPD as a positive control showed similar patterns but with higher responses (Figure 6B) whereas all conditions without antigen showed similar low levels of IL-2, proliferation, and IFN- $\gamma$ production (Figure 6C). This indicates that the observed increased response to CII and PPD was antigen dependent, but not restricted to CII.

These experiments were performed in parallel with experiments on naive mouse spleen cells depleted for T cells, allowing both macrophages and DCs to be present in a ratio as occurring in vivo. This setup allowed us to determine whether the suppressive effect of macrophages overshadows the activating potential of DCs as pro- 
A
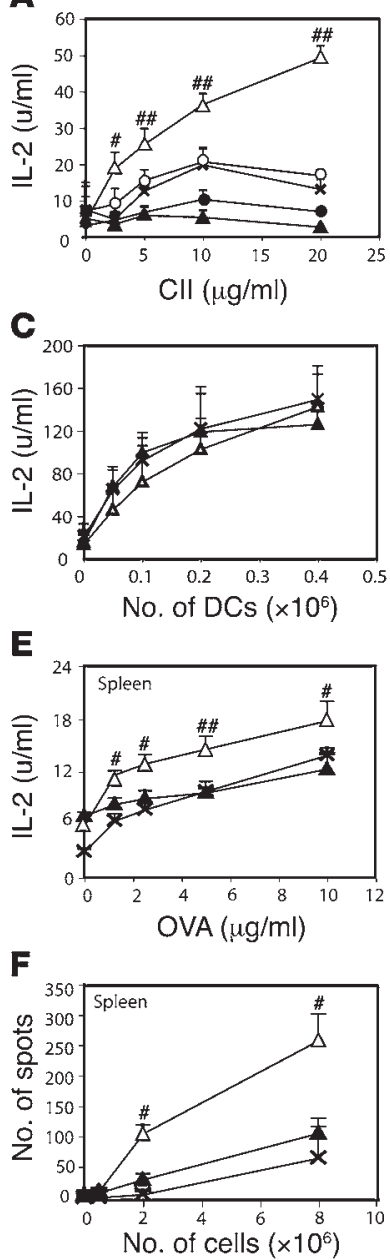

B

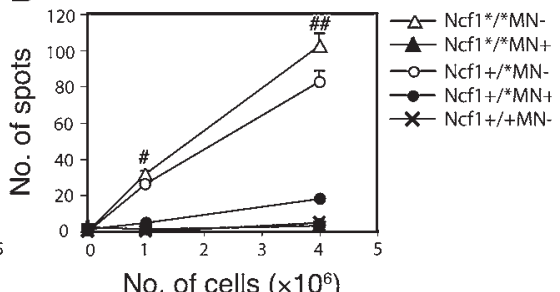

D
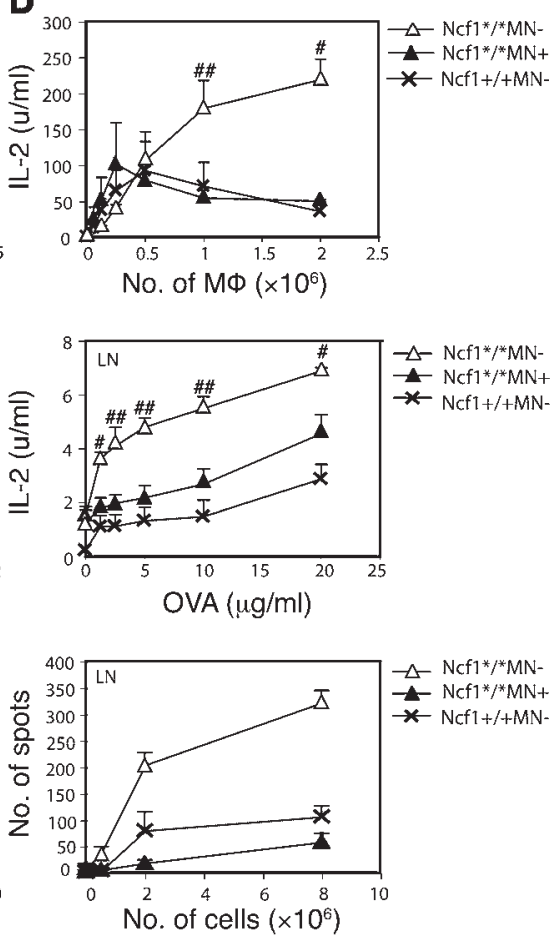

\section{Figure 5}

Mice with mutated Ncf1 in macrophages have more and more active antiCII T cells. Spleen cells from B10.Q ${ }^{M N}$ mice immunized 10 days earlier with CII were restimulated with lathCII, and levels of IL-2 present in the supernatant were measured by ELISA. (A) Ncf1 mutant (*/*) and heterozygous $\left(+{ }^{*}\right)$ transgene-negative mice $\left(\mathrm{MN}^{-}\right)$produced significantly more IL-2 compared with transgene-positive $\left(\mathrm{MN}^{+}\right)$mice. (B) Cells from the same spleens were subjected to IFN- $\gamma$ ELISPOT. The number of spots after restimulation with lathCII was significantly lower in transgenic mice. (C) DCs grown from bone marrow with GM-CSF and matured with LPS induced similar amounts of IL-2 production by HCQ10 hybridoma T cells, irrespective of transgene or Ncf1 genotype. (D) In contrast, p-macrophages (pM $\phi)$ from $\mathrm{Ncf1}^{* / *} \mathrm{MN}^{+}$and

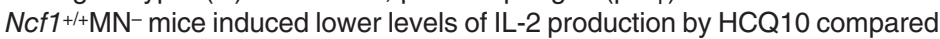
with $\mathrm{Ncf1}^{* / *} \mathrm{MN}-$ mice. IL-2 (E) and IFN- $\gamma(\mathbf{F})$ production after immunization and in vitro restimulation with OVA was higher in $\mathrm{Ncf1}^{* / *} \mathrm{MN}^{-}$mice compared with $\mathrm{Ncf1}^{* / *} \mathrm{MN}+$ and $\mathrm{Ncf1}^{+/+}$mice in both spleen and LNs. For A, B, E, and F, means \pm SEM of 2 experiments with 3 mice per group are shown. $\mathbf{C}$ and $\mathbf{D}$ show the results of 1 out of 2 representative experiments each with 4 mice per group. ${ }^{P}<0.05$; $\# P<0.01$.

fessional APCs. We observed patterns similar to those in Figure 6 for IL-2 and IFN- $\gamma$ production as well as for proliferative responses, suggesting that the suppressive effect of Ncf1-sufficient macrophages is dominant over the T cell-activating effect of Ncf1 mutant DCs.

$T$ cell number and phenotype do not differ between genotypes. The results as shown in Figures 5 and 6 leave room for the possibility that $\mathrm{T}$ cells from $\mathrm{Ncf1}^{* / *} \mathrm{MN}^{-}$mice have higher basal activation levels as compared with $\mathrm{Ncf1}^{* / *} \mathrm{MN}^{+}$or $\mathrm{Ncf1}^{+/+} \mathrm{MN}^{-}$mice. To investigate this, we measured $\mathrm{T}$ cell numbers $\left(\mathrm{CD}^{+}{ }^{+} \mathrm{CD} 4^{+}\right.$ and $\mathrm{CD}^{+}{ }^{+} \mathrm{CD} 8^{+}$) and expression of activation markers (CD44 and CD69) on T cells from naive mice or mice immunized 10 days previously. This was done for blood, spleen, thymus, and LNs (the latter only from immunized mice). No differences were found in CD4 or CD8 T cell numbers (shown as $\mathrm{CD} 4 / \mathrm{CD} 8$ ratio; Figure $7 \mathrm{~A}$ ) nor in expression levels of CD44 and CD69 in naive or immunized mice (Supplemental Figure 2A). To determine whether there was a difference in activation threshold of $\mathrm{T}$ cells from the different genotypes, spleen cells were stimulated with platebound anti-CD3, anti-CD3 plus soluble anti-CD28, concanavalin A (ConA), or PMA in decreasing concentrations. Results from the highest concentrations are shown (anti-CD3, $10 \mu \mathrm{g} / \mathrm{ml}$; anti-CD28, $2 \mu \mathrm{g} / \mathrm{ml}$; ConA, $3 \mu \mathrm{g} / \mathrm{ml}$;PMA, $50 \mathrm{ng} / \mathrm{ml}$ ). We measured T cell-specific cytokines IL-2 and IFN- $\gamma$ and proliferation after 24 hours and 3 days, respectively. No differences in IL-2 production were observed between genotypes (Figure 7). However, IFN- $\gamma$ production was significantly higher in spleens from $\mathrm{Ncfl}^{* / *} \mathrm{MN}^{-}$mice as compared with the other genotypes (Figure $7 \mathrm{C}$ ), similar to the results shown in Figure 5, indicating differences in education. The relatively low levels of IFN- $\gamma$ upon stimulation with ConA or PMA might be due to the fact that total spleen cells were used in contrast with anti-CD3 stimulation, which is $\mathrm{T}$ cell specific. The contrasting high levels of proliferation due to ConA and PMA might be due to proliferation of other cell types as well. The low level of proliferation of $\mathrm{Ncfl}^{* / *} \mathrm{MN}^{-}$ splenocytes upon anti-CD3/28 stimulation might be due to exhaustion of medium due to exaggerated proliferation or another unknown parameter; IL-2 levels are high, as expected, and normally we observe that proliferation follows IL-2 levels. In lower concentrations of anti-CD28 this difference was not as pronounced.

When spleen cells from naive mice were analyzed for IL-2 or IFN- $\gamma$ production upon stimulation with CII, only background levels of these cytokines were observed, and no differences between groups were present (not shown). This confirms that the increased activity of T cells in $\mathrm{Ncfl}^{*} /{ }^{* *} \mathrm{MN}^{-}$ mice is only detectable after antigenic stimulation and not a general phenomenon.

In addition, differences in expression levels of MHC class II and costimulatory molecules on macrophages from the different mice might affect $\mathrm{T}$ cell activation. Expression levels of MHC class II, ICOSL (CD275), CD80, and CD86 were determined by flow cytometry on macrophages $\left(\mathrm{F} 4 / 80^{+} \mathrm{CD} 11 \mathrm{c}^{-}\right)$ from different immune compartments from naive mice or mice immunized 10 days previously, but no differences were observed (Supplemental Figure 2B).

$R O S$ production by macrophages reduces the Th1 response. The high IFN- $\gamma$ and IL-2 responses suggest a predominant Th1 phenotype in the $\mathrm{Ncf}^{*}{ }^{* *} \mathrm{MN}^{-}$mice. To investigate this in further detail, production of other cytokines was determined as well. Spleen cells from $\mathrm{Ncfl}^{* / *} \mathrm{MN}^{-}, \mathrm{Ncfl}^{* / *} \mathrm{MN}^{+}$, and $\mathrm{Ncfl}^{+/+} \mathrm{MN}^{-}$mice immunized 10 days previously were incubated with lathCII, and cytokines were measured in the supernatants after 48 hours. It was observed that levels of IL-2 and IFN- $\gamma$ as well as TNF- $\alpha$ were higher in $\mathrm{Ncfi}^{* / *} \mathrm{MN}^{-}$mice as compared with the other groups (Figure 8). Interestingly, when measuring IL-4 levels, it was found that IL-4 was high- 
A
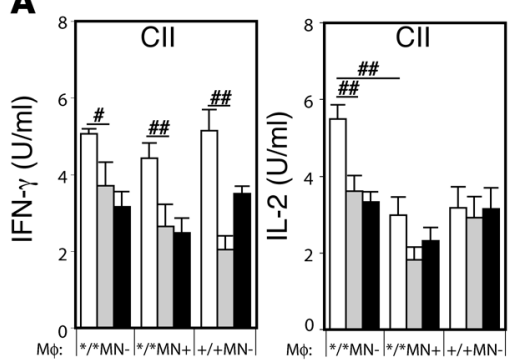

B
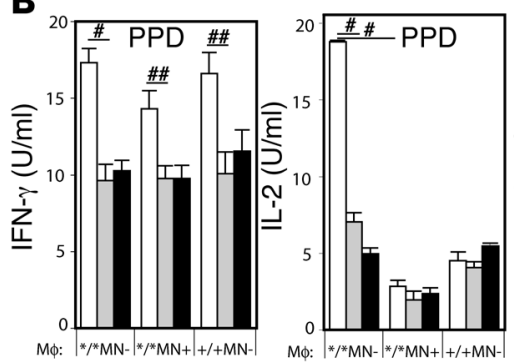

C
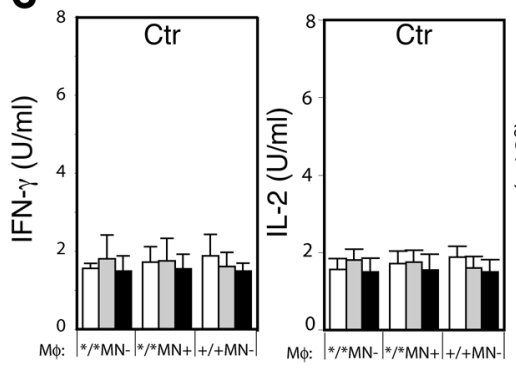
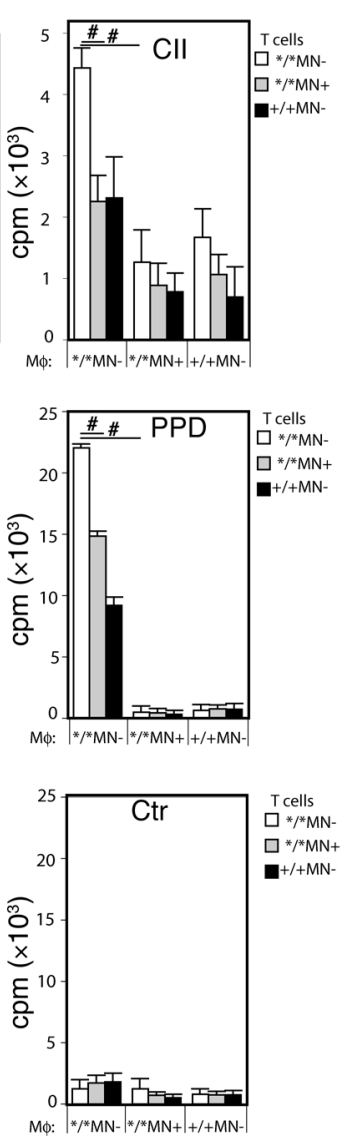

er in spleen cultures from $\mathrm{Ncfl}^{+/+}$mice as compared with $\mathrm{Ncf1}^{* / *} \mathrm{MN}$ mice but also compared with $N c f^{* / *} \mathrm{MN}^{+}$mice. Apparently, cells other than macrophages determined the IL-4 response although it was influenced by the Ncf1 mutation, which was confirmed when measuring IL-4 in the supernatants of the experiments as shown in Figure 6; no differences between genotypes were observed (not shown). For IL-5, no differences between the groups were observed (Figure 8). For IL-10, there was a tendency for $\mathrm{Ncfl}^{* / *}$ mice, irrespective of being transgenic, to produce higher levels of IL-10 as compared with $\mathrm{Ncf1}^{+/+}$mice, although this was not significant with the number of mice used. IL-17 has been shown to play a proinflammatory role in arthritis (21) and might thus be higher in $\mathrm{Ncf1}^{*} /{ }^{*} \mathrm{MN}^{-}$mice, but we could not detect IL-17 in any of the supernatants. These results indicate that mice that lack ROS production in macrophages have a more pronounced Th1 response as compared with mice expressing functional Ncf1 in macrophages, although no obvious Th2 skewing was observed in mice with $\mathrm{Ncf1}$-sufficient macrophages.

\section{Discussion}

Our previous finding that a decreased capacity to exert oxidative burst due to mutations in Ncf1 enhances activation of autoreactive $\mathrm{T}$ cells and the development of arthritis suggests a role for ROS in antigen presentation, especially since we could not find NADPH oxidase-dependent ROS production in T cells $(4,12,13)$. We now show that $\mathrm{Ncf1}$-expressing macrophages mediate protection against arthritis by producing ROS that downregulate $\mathrm{T}$ cell activation. We show that this effect is antigen dependent but not CII specific and is mediated during antigen presentation.

\section{Figure 6}

Macrophage ROS suppress IL-2 but not IFN- $\gamma$ production by Ncf1 mutant T cells. Purified CD4+ T cells from Cll immunized $\mathrm{Ncf1}^{* / *} \mathrm{MN}-$ (white bars), $\mathrm{Ncf1}^{1^{* /} \mathrm{MN}+}$ (gray bars), and $\mathrm{Ncf1}^{+/+\mathrm{MN}-}$ (black bars) were incubated with purified $p$-macrophages from naive mice from the same genotypes (depicted on the $x$ axes) in all 9 combinations and restimulated with lathCII (A), PPD (B), or nothing (Ctr) (C). IFN- $\gamma$ production, IL-2 production, and the proliferative response were determined. IFN- $\gamma$ production was increased in all conditions with $\mathrm{Ncf}^{* / *} \mathrm{MN}^{-} \mathrm{T}$ cells whereas IL-2 production and proliferation were suppressed by macrophages originating from $\mathrm{Ncf}^{* / *} \mathrm{MN}^{+}$or $\mathrm{Ncf}^{+/+} \mathrm{MN}^{-}$mice. Means $\pm \mathrm{SEM}$ of 6-8 mice per group obtained from 3 different experiments are shown. ${ }^{\#} P<0.05,{ }^{\#} P<0.01$.

These findings add a new and important clue to the emerging view that ROS are instrumental in regulating cellular functions $(22,23)$. The production of ROS has most commonly been associated with oxidative stress, which is characterized by a major shift in the cellular redox balance and usually accompanied by ROS-mediated damage (24). However, ROS can also induce changes in the redox balance to regulate cellular activity (25). Redox regulation involves subtle oxidations that might involve modifications in proteins by, for example, modifying cysteine cross-linking and thereby regulate protein function (26-29). T cells are known targets for redox regulation $(14,15)$. It has been reported that exposure of $\mathrm{T}$ cells to ROS downregulates $\mathrm{T}$ cell activity $(12,30,31)$, indicating that $\mathrm{T}$ cells require a reducing milieu for optimal proliferation and activation $(15,30)$. In addition to the extracellular redox level, intracellular redox levels also influence $\mathrm{T}$ cells; a decrease in the intracellular redox balance (oxidation) impairs $\mathrm{T}$ cell function $(22,30)$. In contrast with previous findings $(32,33)$, we did not observe Ncf1 expression or evidence for an induced oxidative burst in $\mathrm{T}$ cells from mice or rats with or without the Ncf1 mutation $(12,13)$. Although we do not exclude production of constitutive low levels of ROS in T cells (34), the very low background DHR123 staining levels did not differ between $T$ cells derived from either strain and thus are Ncf1 independent. This led to the hypothesis that $\mathrm{T}$ cell activation as mediated by ROS is determined by other cells that do produce ROS, like APCs (35). In agreement with established dogma, we found that Ncf1 is not only expressed in neutrophilic granulocytes but also in phagocytic APCs, with the highest expression in macrophages (31).

ROS and the ROS-regulated redox status have been suggested to play a role in antigen presentation; antigen processing by DCs is affected by the number of cysteines and thus disulfide bridges in the antigen (36), and the DC redox status dictates cytokine production (37). In addition, it has been shown that cross-presentation of antigen by NADPH oxidase-deficient DCs is impaired because of decreased $\mathrm{pH}$ in the endosomes, indicating a direct role of ROS in antigen processing (38). Macrophages have been suggested as being more activated in the absence of ROS, as shown in mice with a deficiency of the NADPH oxidase complex (39-41). This was confirmed by a study showing that matrix metalloprotease activity of macrophages was lower in the presence of NADPH oxidase-derived ROS (42). Taken together, it is likely that a change in the redox balance regulated by $\mathrm{NADPH}$ oxidase functionality in APCs affects proteins important for $T$ cell function or uptake and processing of antigen in $\operatorname{APCs}(36,38)$. Indeed, we show here that $\mathrm{T}$ cell activation is determined by levels of ROS produced by macrophages during antigen presentation. Interestingly, Ncf1 expression restricted to macrophages restores $\mathrm{T}$ cell function and arthritis resistance to the level of wild type, suggest- 


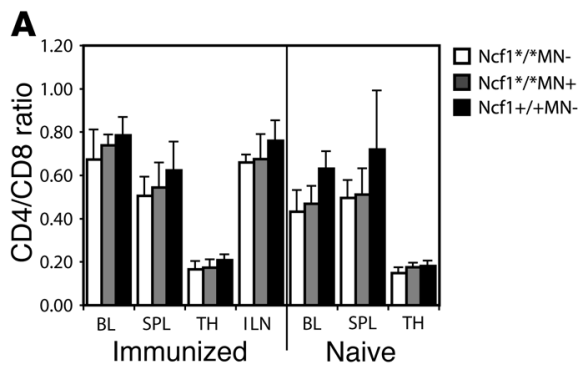

C

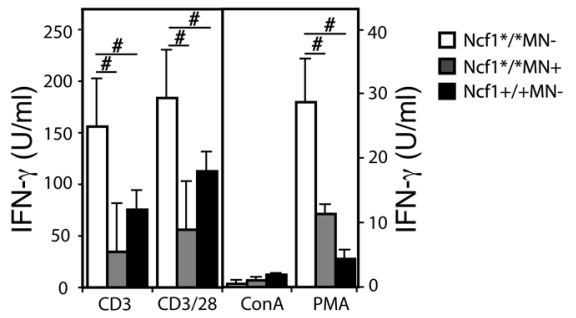

B

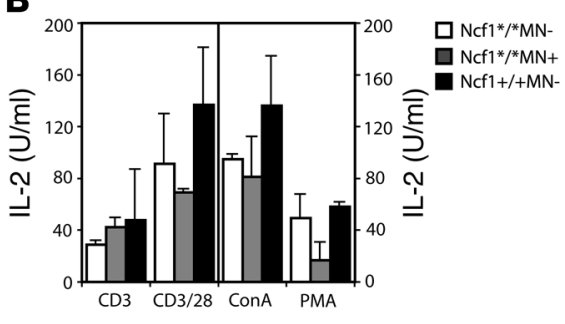

D

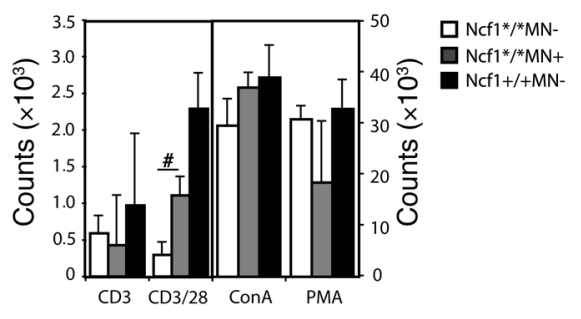

Figure 7

IL-2 production and proliferation do not differ after $\mathrm{T}$ cell stimulation with anti-CD3 or mitogen. (A) $\mathrm{T}$ cell numbers $\left(\mathrm{CD} 3{ }^{+} \mathrm{CD} 4{ }^{+}\right.$ and $\mathrm{CD} 3^{+} \mathrm{CD} 8^{+}$) were measured by flow cytometry in different immune compartments (BL, blood; SPL, spleen; TH, thymus; ILN, inguinal LNs) from naive mice and mice immunized 10 days previously and depicted as CD4/CD8 ratio; there were no differences between the genotypes. (B-D) Stimulation of splenocytes from naive mice with anti-CD3 $(10 \mu \mathrm{g} / \mathrm{ml})$, anti-CD3 plus anti-CD28 $(2 \mu \mathrm{g} / \mathrm{ml})$, ConA $(3 \mu \mathrm{g} / \mathrm{ml})$, or PMA (50 ng/ml) did not result in different levels of IL-2 production or proliferation, but IFN- $\gamma$ production by $\mathrm{Ncf}^{* / *} \mathrm{MN}^{-}$mice was significantly higher compared with $\mathrm{Ncf}^{* / *} \mathrm{MN}^{+}$or $\mathrm{Ncf}^{+/+} \mathrm{MN}^{-}$mice, except for the ConA stimulation. Mean \pm SEM are shown from 4 mice per genotype for all conditions, ${ }^{\#} P<0.05$. ing that macrophages are dominant cells in mediating the oxidative burst effects on $\mathrm{T}$ cell regulation. DCs, in spite of being known as the most professional APCs, did not alter levels of IL-2 production when expressing different $N c f 1$ genotypes different $N c f 1$ genotypes, in contrast to macrophages, and we showed that the macrophage-suppressive effect via ROS was dominant over DC-mediated T cell activation. A possible explanation for this difference is that macrophages are effective in downregulating $\mathrm{T}$ cell responses via ROS whereas DCs that hardly produce ROS rather induce an immune response.

Ncf1 forms a complex with Ncf4 (p40phox) and Ncf2 (p67phox), leading to formation of the NADPH oxidase complex bound to Cybb (gp91phox) and Cyba (p22phox) in the cell membrane. Interestingly, the NADPH oxidase complex is formed in the endosomal/lysosomal membrane and is together with $\mathrm{MHC} /$ peptide complexes transported to the lipid rafts to form the synapse where contacts with the T cell will be made (6). ROS will thus be secreted directly upon the T cell membrane during antigen presentation. APC-derived ROS may not be able to change the intracellular redox balance of $\mathrm{T}$ cells because of their short half-life. However, ROS might affect signal transduction proteins such as LAT or ZAP70 that are located closely beneath the cell membrane $(30,43,44)$ or decrease the number of thiol $(-\mathrm{SH})$ groups on the cell surface and thereby render the $\mathrm{T}$ cells in a less activated state by, e.g., modifying cysteines in the $\mathrm{T}$ cell receptor or costimulatory molecule complex $(12,26)$. This hypothesis is underscored by our observation that naive $N c f 1$ mutant nontransgenic mice have significantly higher levels of T cell surface-SH groups as compared with Ncf1 mutant transgenic mice (not shown). T cells from wild-type rats treated with reduced glutathione to increase the number of cell surface -SH groups transferred PIA in rats (12), but unlike PIA, CIA in mice is not readily transferable by $\mathrm{T}$ cells. However, one can speculate that in mice, macrophage-derived ROS might decrease $\mathrm{T}$ cell activation via this mechanism, which is underscored by the difference in $\mathrm{T}$ cell surface -SH groups that we observed. It is likely that this is determined before immune priming, since the difference was observed in naive mice. Future research should address which proteins are influenced by ROS, resulting in the observed decrease in $\mathrm{T}$ cell activation.

We hypothesize that, by producing ROS, macrophages in central or peripheral lymphoid organs educate $T$ cells to render them less activated in an antigen-dependent fashion in order to prevent (auto- )immune responses in the periphery or to downregulate an ongoing immune response. Indeed, macrophages that could produce ROS suppressed IL-2 production and T cell proliferation in vitro. However, we observed in the crisscross antigen presentation experiments that the IFN- $\gamma$ production by T cells, was dependent on the origin of the $T$ cell and independent of the genotype of the macrophage. This suggests that the IFN- $\gamma$ response, reflecting the activation status of the T cells, is imprinted in vivo and not determined by macrophages during antigen presentation in vitro. Interestingly, stimulation with anti-CD3 or mitogens did not show differences in activation thresholds, suggesting that this imprinting in vivo is antigen dependent. Apart from the observation that ROS influence activation of T cells, it has been shown that the type of $\mathrm{T}$ cell response is also influenced by ROS. As confirmed by our findings, King et al. found that when human $\mathrm{T}$ cells were stimulated in vitro with anti-CD3 and anti-CD28 in presence of ROS, a clear Th2 phenotype was observed in spite of the presence of Th1 cytokines (45). In line with this, it was shown that
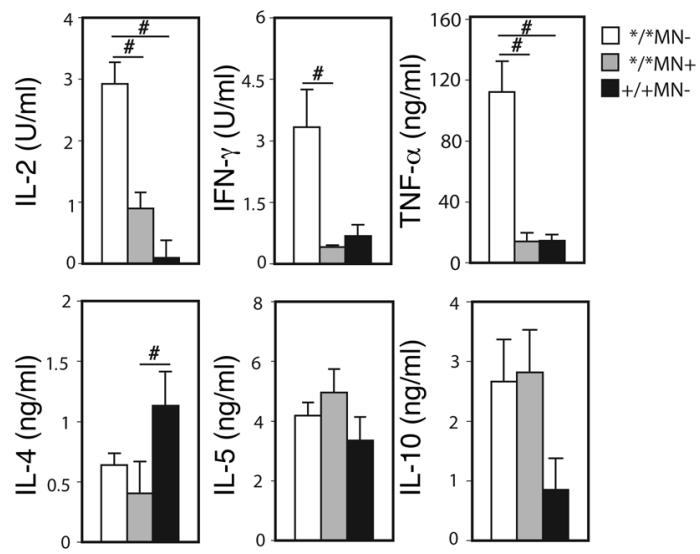

Figure 8

Ncf1 mutated nontransgenic mice have a more pronounced Th1 response. Spleen cells from mice immunized with Cll were restimulated with lathCII in vitro, and levels of IL-2, IFN- $\gamma$, TNF- $\alpha$, IL-4, IL-5, and IL-10 were determined in the supernatant. Mean \pm SEM of $5-6$ mice per group are shown. ${ }^{P} P<0.05$. 
oxidation by depletion of intracellular GSH led to a shift to a Th2 response, characterized by increased IL-4 production and inhibition of IFN- $\gamma$ and IL-12 production $(46,47)$. We now show in vivo that this less pronounced Th1 response in the presence of ROS is mediated via macrophages and that it results in decreased arthritis severity. We could not detect IL-17 in these experiments, but that might be due to a different disease pathogenesis compared with other mouse strains, where IL-17 is the dominant effector cytokine and IFN- $\gamma$ is protective, although this possibility needs further investigation.

Macrophages do not only operate as APCs but are also prominent inflammatory cells in the arthritic joints as has been demonstrated in both CAIA and CIA (46). Interestingly, the Ncf1 mutation did not significantly increase susceptibility to CAIA, excluding an important role for $\mathrm{Ncf} 1$ in macrophages operating in the inflammatory phase.

In conclusion, we have shown that macrophages downregulate $\mathrm{T}$ cell responses by producing ROS, which is mediated during interaction in an antigen-specific way. We provide evidence that $\mathrm{T}$ cells in Ncf1 mutant mice are educated differently and therefore have a different activation status. Ncf1-sufficient macrophages suppress activation of CII T cells in the periphery, which fails in Ncf1 mutant mice, resulting in increased arthritis severity. Based on these findings, we have recently shown that agents activating the NADPH oxidase complex have a therapeutic effect on arthritis in experimental models. These results should lead to reconsideration of the common belief that antioxidants are antiinflammatory.

\section{Methods}

Animals. All mice were genetically controlled and shared the C57BL/10 background in B10.Q mice (originally from J. Klein, Tübingen University, Tübingen, Germany, and maintained in our laboratory for more than 70 generations) expressing the $\mathrm{H} 2-\mathrm{A}^{\mathrm{q}}$ haplotype. Mice with a relative deficiency of $\mathrm{Ncf} 1$ caused by a point mutation $(4,17)$ originated from The Jackson Laboratory and were backcrossed to B10.Q for more than 12 generations. To develop the transgenic mice, the $N c f 1$ coding sequence was amplified from first-strand cDNA, modified to contain cloning sites in the $5^{\prime}$ and $3^{\prime}$ ends. DNA was ligated downstream of the promoter and splice site in a vector containing the human CD68 promoter; the splice signal flanked the first intron and a polyA addition site (16). This construct containing $N c f 1$ was named $M N$. Bacterial sequences were excised and the transgene was introduced into pronuclei from B10.Q. The transgene was crossed to $N c f 1$ mutant B10.Q, creating the B10.Q ${ }^{M N}$ strain; in all experiments, littermates were used. Screening for $N c f 1$ and the MN transgene was performed by PCR, followed by pyrosequence analysis for $N c f 1$, and agarose gel analysis for MN. All animal experiments were approved by the Malmö/Lund ethical committee, Lund, Sweden (license no. M70/04).

CIA and CAIA. CIA was induced by injecting $100 \mu \mathrm{g}$ of rat CII emulsified in CFA intradermally at the base of the tail (4). After 35 days, mice were boosted with $50 \mu \mathrm{g}$ of $\mathrm{CII}$ in incomplete Freund's adjuvant. Arthritis development was scored using a macroscopic scoring system: 1 point was given for each swollen or red toe or joint and 5 points for a swollen ankle, adding up to a maximum score of 60 points per mouse. Serum was taken at day 10, day 42 , and when sacrificed. CAIA was induced by i.v. injection of $4 \mathrm{mAb}$ directed against different epitopes on CII (C1,J1, D3, and U1) with a total amount of $4 \mathrm{mg}$. At day 7 , $25 \mu \mathrm{g}$ of LPS was injected i.p., and disease was followed over time (18).

Antibodies and antigens. The following antibodies were used: F4/80-bio directed against macrophages, N4.18-APCs against CD11c, RA3-6B2cychrome directed against CD45R/B220, M1/70-FITC directed against CD11b, Rb6-bio against Gr-1, 145-2C11-bio against CD3, H129.9-APCs against CD4, IM7-APCs against CD44, and H1.2F3-APCs against CD69 (all from BD Biosciences - Pharmingen). CII was isolated from Lewis rat chondrosarcoma by pepsin or lathyritic digestion as described before $(48,49)$.
Chicken egg albumin (OVA) was obtained from Sigma-Aldrich, and PPD was obtained from Statens Serum Institut.

Flow cytometry. We stained $15 \mu \mathrm{l}$ of blood or cell suspension for Ncf1 or burst as described before $(12,13)$. FcR interaction was blocked by incubation in $5 \%$ serum before and during the first antibody incubation. We analyzed $1 \times 10^{5}-5 \times 10^{5}$ cells with a FACSort, and fluorescence was expressed as GeoMeanX (BD). Mouse anti-human Ncf1 cross-reacting with mouse Ncf1 (clone D-10; Santa Cruz Biotechnology Inc.) or an isotype-matched irrelevant control antibody was used. Cell surface -SH groups were detected with Alexa Fluor 488 or 633 coupled to maleimide (Invitrogen) as described previously $(12,22)$.

Tcell activation assays. Spleens and LNs from naive mice or mice immunized 10 days previously were conferred to cell suspensions. Cells from immunized mice were incubated with lathCII or OVA at different concentrations of antigen or cells. We isolated p-macrophages by injecting $5 \mathrm{ml}$ of ice-cold DMEM into the peritoneal cavity of naive mice; no thioglycollate was used. We depleted p-macrophages from B cells and neutrophils by MACS (Miltenyi); purity was higher than $98 \%$. DCs were grown from bone marrow by stimulation with GM-CSF, as described before (19). DCs were matured with LPS for the last 24 hours. CII was provided for 48 hours, adding ${ }^{3} \mathrm{H}$-thymidine to allow phagocytosis. APCs were coincubated with HCQ10 hybridoma T cells, recognizing $\mathrm{CII}$ in $\mathrm{H} 2$-Aq and CII. After 24 hours, supernatant was assayed for IL-2 by sandwich ELISA. For the crisscross experiments (Figure 6), $\mathrm{T}$ cells were isolated from spleens by negative selection by MACS; spleen cells were - after lysing erythrocytes - incubated with biotinylated Ab directed against DCs, macrophages, granulocytes, NK cells, B cells, and CD8. After incubation with streptavidin-magnetic beads (Miltenyi Biotec), cells were separated twice on a magnetic column. The cell suspensions consisted of over $90 \% \mathrm{CD}^{+} \mathrm{T}$ cells and less than $0.4 \%$ of $\mathrm{CD} 11 \mathrm{~b}^{+}$cells. The remaining $10 \%$ were negative for all markers and seemed to represent erythrocytes on FSC/SSC. Spleen APCs were obtained by depleting for T cells by MACS (anti-CD3/4/8). Twenty-four-hour supernatants were assayed for IL-2; after 72 hours, cells were pulsed with ${ }^{3} \mathrm{H}$-thymidine for $15-18$ hours. Supernatants were then tested for IFN- $\gamma$ production. For experiments shown in Figure 7 , anti-CD3 was used platebound $\left(2\right.$ hours at $\left.37^{\circ} \mathrm{C}\right)$ with or without soluble anti-CD28 (BD Biosciences - Pharmingen).

ELISAs. Serum levels of anti-CII IgG were detected by ELISA as described before (4). As a standard, purified anti-CII Ab from immunized DBA/1 mice was used with known concentration. ELISAs to detect TNF- $\alpha$ and IFN- $\gamma$ were performed in a similar way, using $\mathrm{mAb}$ from BD Biosciences Pharmingen or a kit from Pierce Biotechnology (Endogen; TNF- $\alpha$ ). ELISAs to detect IL-4, IL-2, IL-5, IL-10, and IL-17 were developed using streptavidin-europium and enhancement buffer (PerkinElmer). Ab and standards were obtained from BD Biosciences - Pharmingen.

ELISPOT assays. After prewetting with ethanol, ELISPOT plates were coated with anti-IFN- $\gamma \mathrm{Ab}$, and spleen or LN cells from immunized mice were divided over the plates in different cell concentrations or at 1 cell concentration of $10^{6}$ cells, with different concentrations of lathCII. After 24 hours, cells were decanted and IFN- $\gamma$ was detected with biotinylated antiIFN- $\gamma$, which was detected with streptavidin-labeled alkaline phosphatase. Spots were developed with BCIP/NBT (Sigma-Aldrich). Number of spots was determined with an ImmunoScan ELISPOT reader and analyzed with ImmunoSpot software (Cellular Technology Ltd).

Statistics. For all statistical testing, Mann-Whitney $U$ test was used. $P<0.05$ was regarded as significant. Mean values \pm SEM are shown in all figures.

\section{Acknowledgments}

We thank our animal technicians Carlos Palestro and Rebecca Lindqvist; David Greaves, Oxford University, for the promoter construct; the Anna Greta Crafoord Stiftelse för Reumatologisk 
Forskning, Konung Gustaf V:s 80-års and Wenner Gren Foundation; the EU (MUGEN LSHG-CT-2005-005203, EURAPS LSHM-CT2005-005223, Neuropromise LSHM-CT-2005-018637) and SSF for grants; and Leendert A. Trouw for carefully reading the manuscript.

Received for publication February 23, 2007, and accepted in revised form July 10, 2007.

1. Feldmann, M., Brennan, F.M., and Maini, R.N. 1996. Rheumatoid arthritis. Cell. 85:307-310.

2. MacGregor, A.J., et al. 2000. Characterizing the quantitative genetic contribution to rheumatoid arthritis using data from twins. Arthritis Rheum. 43:30-37.

3. Johannesson, M., Hultqvist, M., and Holmdahl, R. 2006. Genetics of autoimmune diseases: a multistep process. Curr. Top. Microbiol.Immunol. 305:259-276.

4. Hultqvist, M., et al. 2004. Enhanced autoimmunity, arthritis, and encephalomyelitis in mice with a reduced oxidative burst due to a mutation in the Ncf1 gene. Proc. Natl. Acad. Sci. U. S. A 101:12646-12651.

5. Olofsson, P., Holmberg, J., Tordsson, J., Lu, S., Akerstrom, B., and Holmdahl, R. 2003. Positional identification of $\mathrm{Ncf} 1$ as a gene that regulates arthritis severity in rats. Nat. Genet. 33:25-32.

6. Babior, B.M. 1999. NADPH oxidase: an update. Blood. 93:1464-1476.

7. Heyworth, P.G., Cross, A.R., and Curnutte, J.T. 2003. Chronic granulomatous disease. Curr. Opin. Immunol. 15:578-584.

8. Pollock, J.D., et al. 1995. Mouse model of X-linked chronic granulomatous disease, an inherited defect in phagocyte superoxide production. Nat.Genet. 9:202-209.

9. Hitchon, C.A., and El Gabalawy, H.S. 2004. Oxidation in rheumatoid arthritis. Arthritis Res.Ther. 6:265-278.

10. Ghezzi, P., Bonetto, V., and Fratelli, M. 2005. Thioldisulfide balance: from the concept of oxidative stress to that of redox regulation. Antioxid. Redox. Signal. 7:964-972.

11. Reyes, B.M., Danese, S., Sans, M., Fiocchi, C., and Levine, A.D. 2005. Redox equilibrium in mucosal T cells tunes the intestinal TCR signaling threshold. J. Immunol. 175:2158-2166.

12. Gelderman, K.A., Hultqvist, M., Holmberg, J., Olofsson, P., and Holmdahl, R. 2006. T cell surface redox levels determine $\mathrm{T}$ cell reactivity and arthritis susceptibility. Proc. Natl. Acad. Sci. U. S. A. 103:12831-12836.

13. Hultqvist, M., Olofsson, P., Gelderman, K.A., Holmberg, J., and Holmdahl, R. 2006. A new arthritis therapy with oxidative burst inducers. PLoS. Med. 3:e348.

14. Gmunder, H., Eck, H.P., Benninghoff, B., Roth, S., and Droge, W. 1990. Macrophages regulate intracellular glutathione levels of lymphocytes. Evidence for an immunoregulatory role of cysteine. Cell Immunol. 129:32-46.

15. Angelini, G., et al. 2002. Antigen-presenting dendritic cells provide the reducing extracellular microenvironment required for $\mathrm{T}$ lymphocyte activation. Proc. Natl. Acad. Sci. U. S. A. 99:1491-1496.

16. Gough, P.J., Gordon, S., and Greaves, D.R. 2001. The use of human CD68 transcriptional regulatory sequences to direct high-level expression of class A scavenger receptor in macrophages in vitro and in vivo. Immunology. 103:351-361.

17. Huang, C.K., Zhan, L., Hannigan, M.O., Ai, Y., and Leto, T.L. 2000. P47(phox)-deficient NADPH oxidase defect in neutrophils of diabetic mouse Methods. 223:77-92.
Address correspondence to: Rikard Holmdahl, Medical Inflammation Research, I11 BMC, Lund University, S-22184 Lund, Sweden. Phone: 46-46-2224607 ext. 3339; Fax: 46-46-2223110; E-mail: rikard.holmdahl@med.lu.se.

Kyra A. Gelderman's present address is: Department of Nephrology, Leiden University Medical Center, Leiden, The Netherlands. strains, C57BL/6J-m db/db and db/+.J. Leukoc. Biol. 67:210-215.

18. Nandakumar, K.S., and Holmdahl, R. 2005. Efficient promotion of collagen antibody induced arthritis (CAIA) using four monoclonal antibodies specific for the major epitopes recognized in both collagen induced arthritis and rheumatoid arthritis. J. Immunol. Methods. 304:126-136.

19. Lutz, M.B., et al. 1999. An advanced culture method for generating large quantities of highly pure dendritic cells from mouse bone marrow. J. Immunol.

20. Michaelsson, E., et al. 1994. T cell recognition of carbohydrates on type II collagen. J. Exp. Med. 180:745-749.

21. Rohn, T.A., et al. 2006. Vaccination against IL-17 suppresses autoimmune arthritis and encephalomyelitis. Eur. J. Immunol. 36:2857-2867.

22. Sahaf, B., Heydari, K., Herzenberg, L.A., and Herzenberg, L.A. 2003. Lymphocyte surface thiol levels. Proc. Natl. Acad. Sci. U. S. A. 100:4001-4005.

23. Cross, J.V., and Templeton, D.J. 2006. Regulation of signal transduction through protein cysteine oxidation. Antioxid. Redox. Signal. 8:1819-1827.

24. Valko, M., et al. 2007. Free radicals and antioxidants in normal physiological functions and human disease. Int. J. Biochem. Cell Biol. 39:44-84.

25. Saran, M. 2003. To what end does nature produce superoxide? NADPH oxidase as an autocrine modifier of membrane phospholipids generating paracrine lipid messengers. Free Radic. Res. 37:1045-1059.

26. Hogg, P.J. 2003. Disulfide bonds as switches for protein function. Trends Biochem. Sci. 28:210-214.

27. Sahaf, B., Heydari, K., Herzenberg, L.A., and Herzenberg, L.A. 2005. The extracellular microenvironment plays a key role in regulating the redox status of cell surface proteins in HIV-infected subjects. Arch. Biochem. Biophys. 434:26-32.

28. Laragione, T., et al. 2003. Redox regulation of surface protein thiols: identification of integrin alpha4 as a molecular target by using redox proteomics. Proc. Natl. Acad. Sci. U. S. A. 100:14737-14741.

29. DeYulia, G.J., Jr., Carcamo, J.M., Borquez-Ojeda, O., Shelton, C.C., and Golde, D.W. 2005. Hydrogen peroxide generated extracellularly by receptorligand interaction facilitates cell signaling. Proc. Natl. Acad. Sci. U. S. A. 102:5044-5049.

30. Gringhuis, S.I., et al. 2000. Displacement of linker for activation of $\mathrm{T}$ cells from the plasma membrane due to redox balance alterations results in hyporesponsiveness of synovial fluid $\mathrm{T}$ lymphocytes in rheumatoid arthritis. J. Immunol. 164:2170-2179.

31. Forman, H.J., and Torres, M. 2001. Signaling by the respiratory burst in macrophages. IUBMB Life. 51:365-371.

32. Jackson, S.H., Devadas, S., Kwon, J., Pinto, L.A., and Williams, M.S. 2004. T cells express a phagocytetype NADPH oxidase that is activated after T cell receptor stimulation. Nat. Immunol. 5:818-827.

33. Gulow, K., et al. 2005. HIV-1 trans-activator of transcription substitutes for oxidative signaling in activation-induced T cell death. J. Immunol. 174:5249-5260.
34. Remans, P.H., et al. 2005. Intracellular free radical production in synovial $\mathrm{T}$ lymphocytes from patients with rheumatoid arthritis. Arthritis Rheum. 52:2003-2009.

35. Matsue, H., et al. 2003. Generation and function of reactive oxygen species in dendritic cells during antigen presentation. J. Immunol. 171:3010-3018.

36. Li, P., Haque, M.A., and Blum, J.S. 2002. Role of disulfide bonds in regulating antigen processing and epitope selection. J. Immunol. 169:2444-2450.

37. Murata, Y., Ohteki, T., Koyasu, S., and Hamuro, J. 2002. IFN-gamma and pro-inflammatory cytokine production by antigen-presenting cells is dictated by intracellular thiol redox status regulated by oxygen tension. Eur. J. Immunol. 32:2866-2873.

38. Savina, A., et al. 2006. NOX2 controls phagosomal $\mathrm{pH}$ to regulate antigen processing during crosspresentation by dendritic cells. Cell. 126:205-218.

39. Snelgrove, R.J., Edwards, L., Rae, A.J., and Hussell, T. 2006. An absence of reactive oxygen species improves the resolution of lung influenza infection. Eur. J. Immunol. 36:1364-1373.

40. Komatsu, J., Koyama, H., Maeda, N., and Aratani, Y. 2006. Earlier onset of neutrophil-mediated inflammation in the ultraviolet-exposed skin of mice deficient in myeloperoxidase and NADPH oxidase. Inflamm. Res. 55:200-206.

41. Snelgrove, R.J., Edwards, L., Williams, A.E., Rae, A.J., and Hussell, T. 2006. In the absence of reactive oxygen species, $T$ cells default to a Th1 phenotype and mediate protection against pulmonary Cryptococcus neoformans infection. J. Immunol. 177:5509-5516.

42. Kassim, S.Y., et al. 2005. NADPH oxidase restrains the matrix metalloproteinase activity of macrophages. J. Biol. Chem. 280:30201-30205.

43. Gringhuis, S.I., Breedveld, F.C., and Verweij, C.L. 2002. Linker for activation of T cells: sensing redox imbalance. Methods Enzymol. 352:248-257.

44. Gringhuis, S.I., et al. 2002. Effect of redox balance alterations on cellular localization of LAT and downstream T-cell receptor signaling pathways. Mol. Cell. Biol. 22:400-411.

45. King, M.R., Ismail, A.S., Davis, L.S., and Karp, D.R. 2006. Oxidative stress promotes polarization of human $\mathrm{T}$ cell differentiation toward a $\mathrm{T}$ helper 2 phenotype. J. Immunol. 176:2765-2772.

46. Solomon, S., Rajasekaran, N., Jeisy-Walder, E., Snapper, S.B., and Illges, H. 2005. A crucial role for macrophages in the pathology of K/B x N seruminduced arthritis. Eur. J. Immunol. 35:3064-3073.

47. Peterson, J.D., Herzenberg, L.A., Vasquez, K., and Waltenbaugh, C. 1998. Glutathione levels in antigen-presenting cells modulate Th1 versus Th2 response patterns. Proc. Natl. Acad. Sci. U. S. A. 95:3071-3076

48. Andersson, M., and Holmdahl, R. 1990. Analysis of type II collagen-reactive T cells in the mouse. I. Different regulation of autoreactive vs. non-autoreactive anti-type II collagen T cells in the DBA/1 mouse. Eur. J. Immunol. 20:1061-1066.

49. Miller, E.J., and Rhodes, R.K. 1982. Preparation and characterization of the different types of collagen. Methods Enzymol. 82:33-64. 